
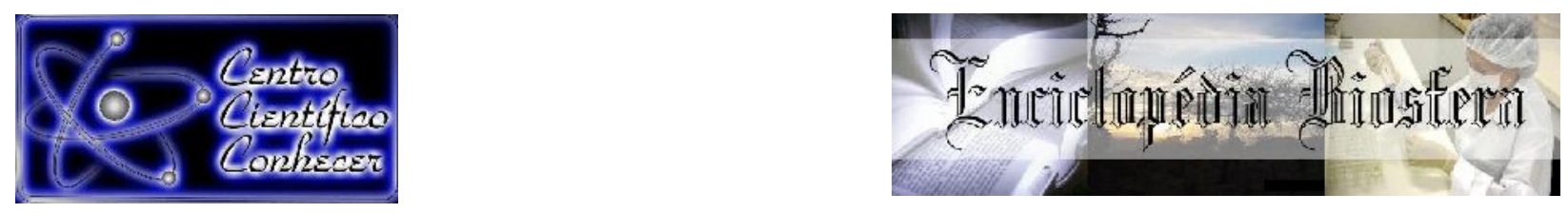

\title{
EDUCAÇÃO EM SAÚDE: VISITA DOMICILIAR E AVALIAÇ̃̃O EPIDEMIOLÓGICA EM PARCERIA COM O PROGRAMA DE SAÚDE DA FAMÍLIA
}

Taísa Dias Prado ${ }^{1}$, Patrícia Pereira Azevedo², Vanessa Borges Almeida², Marcos Michael da Silva ${ }^{1}$ e Heliana Batista de Oliveira ${ }^{3}$

${ }^{1}$ Bacharel em Ciências Biológicas pela Universidade Federal de Goiás - Regional Catalão

${ }^{2}$ Bacharel em Enfermagem pela Universidade Federal de Goiás - Regional Catalão

${ }^{3}$ Professora do Curso de Ciências Biológicas da Universidade Federal de Catalão, Catalão-GO, Brasil.

heliana@ufcat.edu.br

Recebido em: 15/08/2021 - Aprovado em: 15/09/2021 - Publicado em: 30/09/2021

DOI: $10.18677 /$ EnciBio 2021C17

trabalho licenciado sob licença Creative Commons Attribution-NonCommercial-NoDerivatives 4.0 International License.

\section{RESUMO}

O presente trabalho teve como objetivo principal realizar ações de Educação em Saúde, juntamente com o Programa de Saúde da Família, por meio da problematização de assuntos voltados à prevenção de doenças, manutenção e recuperação da saúde local na cidade de Catalão-GO. O estudo consistiu na aplicação de questionários socioepidemiológicos a 30 famílias, com o objetivo de diagnosticar as condições higiênico-sanitárias das mesmas. Os principais resultados mostram que as famílias possuem renda de um salário mínimo, baixo grau de escolaridade e precárias condições higiênico-sanitárias. O estudo evidenciou a necessidade de trabalhos relacionados à promoção da saúde local e a importância da adoção de medidas profiláticas por meio da educação em saúde para melhorar a qualidade de vida da população.

PALAVRAS-CHAVE: Educação em Saúde. Enteroparasitoses. Programa de Saúde da Família.

\section{HEALTH EDUCATION: HOME VISIT AND EPIDEMIOLOGICAL ASSESSMENT IN PARTNERSHIP WITH THE FAMILY HEALTH PROGRAM}

\section{ABSTRACT}

The present work had as main objective to carry out Health Education actions, together with the Family Health Program, through the problematization of issues related to disease prevention, maintenance and recovery of local health in the city of Catalão-GO. The study consisted of the application of socio-epidemiological questionnaires to 30 families, in order to diagnose their hygiene and sanitary conditions. The main results show that families have a minimum wage income, low education level and precarious hygienic-sanitary conditions. The study highlighted the need for work related to local health promotion and the importance of adopting prophylactic measures through health education to improve the population's quality of life.

KEYWORDS: Health Education. Enteroparasitoses. Family Health Program. 


\section{INTRODUÇÃO}

As enteroparasitoses são infecções causadas por helmintos ou protozoários, podendo apresentar no seu ciclo biológico fases parasitárias (hospedeiro definitivo, hospedeiro intermediário e/ou vetor) e fase de vida livre (SHRESTHA et al., 2018). Os helmintos mais frequentemente relacionados a enteroparasitoses são Ascaris lumbricoides, Taenia solium, Trichuris trichiura, Enterobius vermicularis, Strongyloides stercoralis e ancilostomatídeos os quais são transmitidos principalmente por meio de água e alimentos contaminados ou penetração ativa na pele. Dentre os protozoários destacam-se Giardia lamblia e Entamoeba histolytica, sendo a fonte de infecção fecal-oral (DACAL et al., 2020).

Estes parasitas promovem a contaminação e disseminação por meio da eliminação de ovos, larvas ou cistos em conjunto com as fezes, ocasionando a contaminação do ambiente e do solo, podendo os ovos ou cistos ser levados aos alimentos ou arrastados pela água atingindo mananciais utilizados no abastecimento de cidades e irrigação de plantações. Outro modo de contaminação bastante comum por ovos e cistos ocorre pelo contato da mão suja com a boca, em adultos e crianças, chamado de via fecal-oral. Em relação às larvas, a contaminação ocorre pela penetração na pele do hospedeiro (NEVES, 2016).

Essas doenças apresentam elevada prevalência e manifestações clínicas distintas. Além disso, constituem um sério problema de saúde pública, uma vez que aproximadamente um terço da população brasileira encontra-se em condições ambientais inadequadas de higiene e educação. Em suma, devido à precariedade das condições sanitárias, as regiões tropicais e subtropicais configuram-se como os principais focos da doença, tornando-as propícias à disseminação das infecções parasitárias (FELEKE et al., 2019).

Deste modo, a elevada prevalência de infecções parasitárias tem ocasionado como consequência um acréscimo no número de patogenias. A maioria destas enfermidades são quase sempre negligenciadas e esquecidas, uma vez que os sintomas clínicos apresentados pelos pacientes são inespecíficos ou confundidos com os de outras doenças (DACAL et al., 2020). Os pacientes acometidos por tais enfermidades sofrem longos anos de parasitoses, de forma silenciosa, tendo como consequência danos principalmente aos jovens e crianças, que constituem o principal grupo de risco, uma vez que a transmissão, na maioria das infecções, ocorre pela via oral e fecal (SHRESTHA et al., 2018).

A presença de infecção parasitária correlaciona-se com determinantes diversos, os quais contribuem no processo saúde-doença de uma população. Em meio destes é possível ressaltar o saneamento básico precário, a ingestão de água não tratada, a ausência de sistema de esgotamento sanitário, o contato direto e contínuo com o solo, precários hábitos de higiene e o desconhecimento a respeito do mecanismo de infecção, que pode ter relação com o nível cultural dos envolvidos (DACAL et al., 2020).

As doenças parasitárias são preocupantes devido à mortalidade e frequência com que produzem déficits orgânicos, sendo um dos principais fatores debilitantes da população. Sobretudo estas enfermidades encontram-se associadas frequentemente a quadros de diarreia crônica e desnutrição, comprometendo assim, o desenvolvimento físico e intelectual, particularmente das faixas etárias mais jovens da população (MORAES et al., 2019). Vale ressaltar que as infecções intestinais por parasitas têm relação com os padrões inadequados de higiene, sendo a habitação e o peridomicílio os locais que oferecem maior risco de contaminação (SHRESTHA et al., 2018). No Brasil se faz evidente o déficit no setor de saneamento básico, 
sobretudo no que se refere à implantação e tratamento de esgoto, apresentando maior carência nas áreas periféricas dos centros urbanos e nas zonas rurais, onde está concentrada a população mais pobre (GOMES, 2020). Intervenções de saúde pública, como o fornecimento de água potável, atividades de educação em saúde, inspeção da higiene dos alimentos e manutenção dos sistemas de saneamento em funcionamento são essenciais para o controle em longo prazo das enteroparasitoses na comunidade (SHRESTHA et al., 2018).

Partindo como pressuposto a importância do tratamento de água e esgoto no controle de várias doenças, entre estas as enteroparasitoses, torna-se preocupante o fato de que uma em cada três pessoas no mundo não tem acesso a água potável e cerca de 670 milhões de pessoas não utilizam instalações sanitárias residenciais (GOMES, 2020). Especificamente no Brasil, pouco mais de $85 \%$ dos domicílios são abastecidos com água. Esse número pode cair dependendo da região, chegando a $58,8 \%$ na região Norte do País. As regiões Norte e Nordeste do País também são as mais atingidas pela falta de rede de esgoto. Apresentam $27,4 \%$ e $47,2 \%$, respectivamente, de cobertura (IBGE, 2019).

Entre os efeitos negativos causados pela falta de saneamento básico, estão as doenças conhecidas como doenças de transmissão hídrica (gastrenterites agudas, hepatite viral do tipo A e E, parasitoses intestinais), doenças transmitidas por vetores (pediculose, escabiose, dengue, leishmaniose, toxoplasmose e cisticercose), outros agravos indiretamente como desnutrição, doenças e intoxicações por gases e todas essas doenças podem levar a morte especialmente entre crianças que não possuem hábitos de higiene (CONASS, 2016).

Tal situação vinculada às baixas condições socioeconômicas, as diferenças geográficas e climáticas, aos níveis variados de escolaridade, condições de saneamento precário e o crescimento acelerado e não planejado de determinadas cidades, criam zonas periféricas empobrecidas, com moradias constituídas sem a mínima infraestrutura cujas condições favorecem a transmissão de parasitas intestinais (NEVES, 2016). Segundo Halliday et al. (2020) vários fatores estão associados a coinfecção parasitária, dentre eles a estrutura das cidades e aglomerados populacionais.

O equacionamento dessa problemática esbarra na falta de investimentos em obras de saneamento básico e falta de programas educativos capazes de envolver as comunidades para a mudança de hábitos e crenças. Nesse sentido, as parasitoses intestinais constituem um dos melhores exemplos das complexas interrelações entre hospedeiro, agente e ambiente, como causadores e mantenedores de um padrão de infecção de forma endêmica da comunidade e são, nesse contexto, um dos problemas graves que ainda persistem na sociedade brasileira (REY, 2015).

Segundo dados da Organização Mundial de Saúde, essas doenças afetam bilhões de pessoas, levando a óbito, anualmente, outros milhões. É estimado que a cada quatro pessoas, uma encontra-se infectada (WHO, 2020). Ao contrário de outras patogenias, a pré-disposição racial, genética ou a suscetibilidade específica, não demonstram ser os fatores que determinam a prevalência de parasitas intestinais. No entanto, as diferenças referentes ao acesso à educação, a cultura e nos hábitos alimentares podem aumentar a exposição à infecção (DE CARLI et al., 2013).

Outro problema de saúde pública encontrado na população de locais em desenvolvimento são as doenças ectoparasitárias como a pediculose, escabiose e a larva migrans cutânea que estão intimamente ligadas a falta de educação em saúde 
de diferentes comunidades onde programas de controle são escassos ou inexistentes (COATES et al., 2020).

Diante desse contexto a promoção da saúde visa alguns valores como qualidade de vida, saúde, equidade, democracia, cidadania, desenvolvimento, participação e parceria. Além de combinação de estratégias como ações do Estado (políticas públicas saudáveis), da comunidade (reforço da ação comunitária), de cidadãos (desenvolvimento de habilidades pessoais), do sistema de saúde (reorientação do sistema de saúde) e de parcerias intersetoriais (SILVA et al., 2020).

A Promoção da Saúde, a prevenção de doenças e o tratamento e reabilitação das enfermidades são as três principais estratégias para intervir no processo saúdedoença. Essas estratégias encontram-se situadas em campos de conhecimento bastante complexos e exigem esforços integrados para levá-las a melhoria da saúde da população (SILVA et al., 2020).

A socialização de informações é crucial, tendo como contexto principal o enfoque da prevenção, se apresentando com uma das ferramentas potenciais para o controle de doenças parasitárias incluindo medidas simples que levem a melhoria da qualidade de vida. A educação em saúde é uma ferramenta importante que requer investimentos dos líderes governamentais, vontade política, capacitação e incentivo dos profissionais que atuam no sistema público de saúde (MOLYNEUX et al., 2017).

Neste sentido, o presente trabalho objetivou realizar ações de educação em saúde em conjunto com o Programa de Saúde da Família da cidade de Catalão Goiás, por meio da problematização de assuntos voltados à prevenção de doenças, manutenção e recuperação da saúde local, por entender a saúde como um direito inerente ao exercício da cidadania.

\section{Área de estudo}

\section{MATERIAL E MÉTODOS}

O município de Catalão situa-se no sudeste de Goiás, com uma população de 108.823 habitantes. A cidade possui 116 bairros, entre estes o Setor Aeroporto, bairro escolhido para realização do trabalho por ser um bairro de classe média baixa. O Setor Aeroporto compreende também os loteamentos Santa Mônica e São Lucas com uma população de 1.114 habitantes (IBGE, 2019).

\section{População de estudo}

O estudo ocorreu entre os meses de setembro a dezembro de 2013, em parceria com o Programa de Saúde da Família - Unidade Ipanema, juntamente com discentes dos cursos de Ciências Biológicas- Licenciatura/Bacharelado e Enfermagem com visitas ao bairro Setor Aeroporto.

A comunidade deste bairro encontra-se entre as mais propícias a infecções por diversas doenças em virtude das condições de saneamento básico precário. Neste estudo a campo houve o envolvimento social local (interação com Unidades Básicas de Saúde locais e presidente da Associação de Moradores da Comunidade) e o aprofundamento da análise, discussão e reflexão das atividades propostas.

Foi realizada a identificação do conhecimento prévio da comunidade a respeito de doenças infecciosas e parasitoses intestinais por meio de relatos pessoais. Foram também analisadas as ferramentas ideais para pessoas que vivem nas mesmas condições da população observada. 


\section{Coleta de dados}

Foram selecionadas 30 famílias cadastradas para participação da pesquisa e após o contato com a população, explicou-se o processo e os objetivos do trabalho a ser realizado. A obtenção dos dados ocorreu através de uma entrevista, em que os participantes responderam a um questionário, referente ao saneamento básico e qualidade da água de onde vivem e hábitos de higiene, e as variáveis trabalhadas foram: sexo, idade, escolaridade, aspectos socioeconômicos, parasitoses, pediculose. Os entrevistadores tiveram o apoio de um agente de saúde que os acompanharam nas visitas, facilitando o acesso aos domicílios e às famílias.

\section{Aspectos éticos}

Este trabalho foi aprovado pelo Comitê de Ética em Pesquisa - CEP (304/2010), Educação em saúde: visita domiciliar e avaliação epidemiológica em parceria com o Programa de Saúde da Família, o qual está vinculado ao projeto "Estratégias teórico-práticas em Microbiologia na Educação Básica e Superior". Cada participante da pesquisa foi orientado a assinar o Termo de Consentimento Livre e Esclarecido (TCLE), inserindo-se e/ou aceitando sua participação na pesquisa.

\section{Análise de dados}

Quanto à análise estatística, utilizou-se o teste de Qui-Quadrado para comparação da positividade entre as faixas etárias, o período das análises e o gênero. Os dados foram analisados utilizando o software GraphPad Prism versão 3.0. Todos os resultados foram considerados estatisticamente significativos a um nível de significância de $5 \%(p<0,05)$.

\section{RESULTADOS E DISCUSSÃO}

De acordo com a avaliação socioeconômica realizada nas residências, a maioria dos participantes era do sexo feminino (93,3\%), $70 \%$ se encaixavam na faixa etária entre 26 e 39 anos e $70 \%$ trabalhavam apenas no lar. Foi possível perceber que a maioria das famílias apresentou renda de um salário mínimo (63,3\%). Quanto à escolaridade, $50 \%$ disseram ter apenas o ensino fundamental. Nenhum participante cursou ensino superior (Tabela 1).

Segundo Birch (2017) quanto menor o grau de instrução, menor será a contribuição educativa que o indivíduo poderá oferecer ao seu familiar, podendo ainda influenciar nas práticas relacionadas aos cuidados com as mesmas. Acreditase que a educação seja o fator determinante para minimizar os graves problemas de saúde pública, uma vez que a mesma proporciona uma concepção global e integrada do mundo. 
TABELA 1: Características sociodemográficas dos usuários do Programa Saúde da Família- Ipanema.

Variáveis \%

$\begin{array}{lc}\text { Sexo } & \\ \text { Feminino } & 93,3^{* * *} \\ \text { Masculino } & 6,7\end{array}$

\section{Faixa etária (anos)}

$\leq 25$

De 26 a 39

De 40 a 59

\section{Escolaridade}

Analfabeto

Fundamental incompleto

13,3

Fundamental completo

Médio incompleto

13,3

Médio completo

16,7

Superior

\section{Profissão}

Do lar

Aposentados

Doméstica

Outros

\section{Renda mensal}

1 salário

2 a 4 salários

5 a 7 salários

${ }^{* \star *} p<0,0001,{ }^{* *} p<0,01,{ }^{*} p<0,05$

O grande índice de baixa renda familiar encontrado é preocupante, pois a renda é um fator intimamente relacionado com a alimentação da família e com o índice de parasitoses. A baixa renda infere diretamente na nutrição familiar, uma vez que estes indivíduos não apresentam condições financeiras necessárias para realizar uma alimentação de qualidade e, por consequência, podem apresentar déficit de nutrição. Conforme salientado por Moraes et al. (2019) em indivíduos mal nutridos, as parasitoses constituem fatores importantes de anemia e desnutrição em virtude de terem suas reservas orgânicas disputadas pelas necessidades energéticas do indivíduo e pela ação espoliativa dos parasitos.

Quanto ao local de residência dos entrevistados, $73,3 \%$ disseram possuir casa própria, $16,7 \%$ dependem de casas de aluguel e 10\% viviam em casas adquiridas por meio de doação. O número de residentes no domicílio variou entre o valor mínimo de 2 e o máximo de 9 moradores, destacando-se os seguintes percentuais: 3 moradores (26,7\%), 4 moradores (16,7\%), 5 moradores $(30 \%)$ sendo a média de 5 moradores por domicílio. De acordo com as condições habitacionais da população pesquisada, $80 \%$ das casas estavam em condições inacabadas (Tabela 2). 
TABELA 2: Condições de habitação dos domicílios avaliados.

\begin{tabular}{lc}
\hline Variáveis & $\%$ \\
\hline Tipo de residência & $73,3^{* *}$ \\
Casa Própria & 16,7 \\
Alugada & 10 \\
Doada & \\
\hline Estado da residência & 20 \\
Pronta & $80^{* *}$ \\
Inacabada &
\end{tabular}

A moradia configura-se, segundo o conceito de habitação saudável, como um importante indicativo da saúde dos habitantes, bem como se apresenta estreitamente relacionado com o território geográfico e social onde se assenta. Os materiais utilizados para sua construção, a segurança e qualidade dos elementos, o processo construtivo, a composição espacial, a qualidade dos acabamentos, o contexto global do entorno (comunicações, energia, vizinhança) e a educação em saúde e ambiental de seus moradores influencia na condição de vida saudável (SAPS, 2012). Na população estudada, a maior parte das casas eram classificadas como habitáveis, no entanto muitas residências encontravam-se inacabadas.

De acordo com as condições de saneamento, a origem da água utilizada é proveniente da rede pública em $98 \%$ das residências. Quanto ao destino da água e dejetos, $100 \%$ das residências faziam uso de fossa coberta, não apresentando coleta e tratamento de esgoto. A coleta de lixo no bairro ocorre três vezes por semana, mesmo assim os lotes, calçadas e meios-fios apresentavam, em sua maioria, grande quantidade de lixo (Tabela 3).

TABELA 3. Condições de Saneamento básico nos domicílios avaliados.

\begin{tabular}{ll}
\hline Variáveis & $\%$ \\
\hline Origem da água utilizada nos domicílios & \\
Rede Pública & $98^{* * *}$ \\
Nascente/Mina & - \\
Poço comum & 2
\end{tabular}

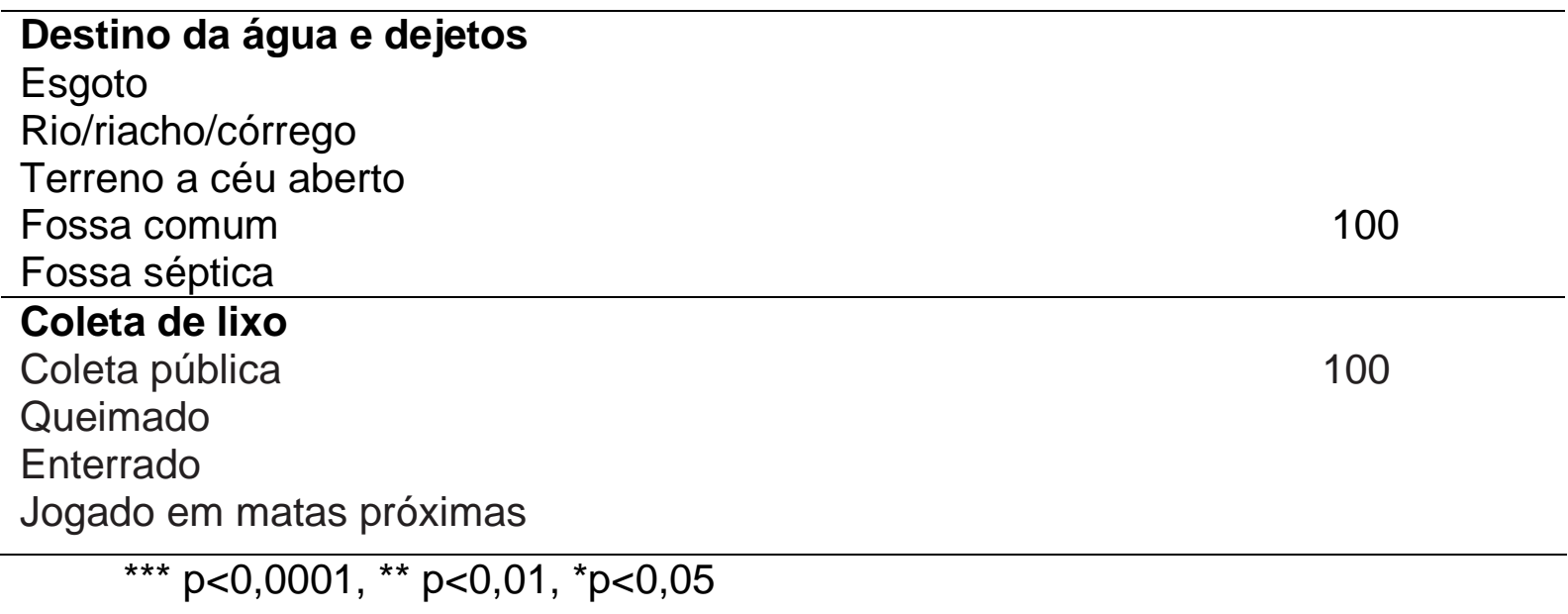


Neste estudo foi observada a ausência de tratamento de esgoto nas residências pesquisadas. Visto a importância do saneamento básico no controle de doenças infecciosas e parasitárias, torna-se imprescindível à presença de meios eficientes de contenção de dejetos. As fossas sépticas podem ser uma solução adequada para o destino dos dejetos sanitários, para tanto é imprescindível que sejam construídas adequadamente com limpeza e manutenção regulares (CHAOUA et al., 2018).

Em relação a higienização, $60 \%$ mostraram ter o hábito de lavar as mãos antes das refeições e 6,7 \% disseram não realizá-la, sendo a maioria crianças. Os moradores da maioria dos domicílios declararam não realizar a preparação correta dos alimentos antes do consumo, representando 26,6\%. Em relação à ingestão de água filtrada, $36,7 \%$ dos participantes visitados relataram realizá-la, enquanto que $63,3 \%$ ingerem água tratada de torneira, sem filtrar. A pediculose foi um problema encontrado em $16 \%$ dos entrevistados, a maioria crianças. Apenas 10,2\% relataram estar parasitados devido a exames realizados recentemente, e os demais não souberam responder, pois não realizavam exames há mais de dois anos. A ingestão de carne bovina foi mais frequente na maioria das residências representando $66,7 \%$, seguindo de aves com $20 \%$ e a carne suína $13,3 \%$.

É importante ressaltar que o consumo de água não filtrada pode afetar a saúde de várias maneiras: pela ingestão direta, na preparação de alimentos, na higiene pessoal, na agricultura, na higiene do ambiente, nos processos industriais ou nas atividades de lazer (FNS, 2019). O perigo à saúde se deve ao fato de que a água pode ser um importante veículo de agentes biológicos e químicos potencialmente nocivos ao homem quando há falta de cuidado e efetivo tratamento, comprometendo assim a saúde e o bem-estar da comunidade (BROWN et al., 2019). Outro fator preocupante é que a principal fonte de infecção de Giardia lamblia ocorre pela ingestão de água não filtrada. A microrregião de Catalão apresenta altos índices desse parasito, principal responsável por diarreias e má absorção intestinal (BORGES, et al., 2011). Diante desses fatores, a educação em saúde exerce importante impacto no bem estar humano, assegurando, a partir de hábitos corretos de higiene que o sistema imunológico dos indivíduos não seja afetado de forma negativa.

TABELA 4. Avaliação do perfil parasitário e hábitos de higiene.

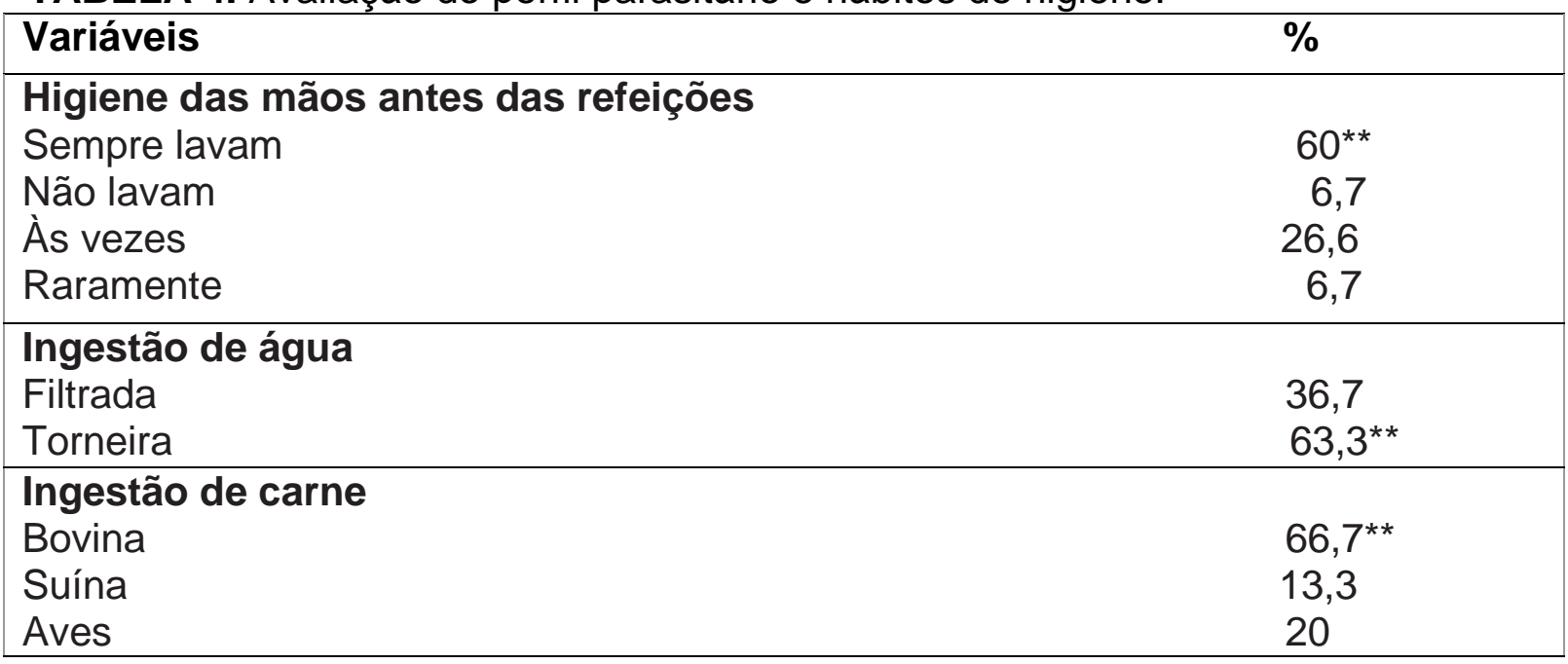




\begin{tabular}{|lc|}
\hline Parasitose & \\
Presente & 10,2 \\
Ausente & - \\
Não sabem & $89,8^{* \star *}$ \\
\hline Pediculose & 16 \\
Presente & $84^{* *}$ \\
Ausente & \\
\hline${ }^{* \star *} \mathrm{p}<0,0001,{ }^{* *} \mathrm{p}<0,01$ & \\
\hline
\end{tabular}

Os parasitos intestinais estão entre os patógenos mais frequentemente encontrados em seres humanos. As enteroparasitoses são consideradas agentes importantes da epidemiologia da desnutrição e da diarreia crônica na infância nos países em desenvolvimento (FELEKE et al., 2019). A porcentagem de enteroparasitoses observada nos questionários não foi significante, pois a maioria não soube relatar sobre uma possível infecção. Embora representem um importante problema de saúde pública nos países subdesenvolvidos, as parasitoses intestinais recebem pouca atenção nas ações dos programas de saúde, em que a alta prevalência de infecções entre as comunidades decorre da elevada contaminação ambiental, o que necessita permanentemente de condições básicas de educação e saneamento básico. Diante do exposto se faz necessário o conhecimento da frequência de enteroparasitoses da população estudada por meio de testes coproparasitológicos, uma vez que o diagnóstico e tratamento de doentes são importantes ferramentas profiláticas.

Em virtude da facilidade de disseminação, a pediculose constitui-se um problema de saúde pública de difícil controle e que requer constante conscientização. A prevalência de pediculose está concentrada em crianças e adolescente que frequentam ambientes escolares, no entanto é importante ressaltar que essa infestação também acomete homens e mulheres na fase adulta (COATES et al., 2020). Ainda que apenas $16 \%$ da população do estudo tenha respondido positivamente para pediculose, segundo os agentes de saúde local, a mesma se apresenta de forma recorrente na região.

A incidência de pediculose assumiu proporções globais, sendo um problema recorrente. Uma vez que tal enfermidade acomete principalmente estudantes, a mesma tende a atrapalhar o rendimento escolar. Isso se deve à diminuição da autoestima e dificuldade de concentração, proporcionado pelo prurido contínuo e distúrbios do sono. Em casos mais graves, devido à hematofagia do piolho, as crianças podem ainda desenvolver anemia (NEVES, 2016).

A qualidade de vida vem sendo discutida por diferentes olhares como critério básico na aplicação de estratégias que propiciem a saúde do homem. Diversas enfermidades que acometem o corpo humano, geralmente, têm o diagnóstico fundamentado pela manifestação de células microbianas patogênicas (MADIGAN et al., 2016) e/ou por proteínas microbianas com efeito tóxico (RAY, 2013). Práticas de higiene pessoal, comportamental e ambiental são fatores essenciais para eliminarem e/ou minimizarem as doenças infecciosas sendo relevante a orientação de indivíduos de diferentes faixas etárias para assegurar tais medidas (BIRCH, 2017).

A Carta de Ottawa, gerada na I Conferência Internacional sobre Promoção da Saúde, de 1986 representa o principal conceito para a promoção da saúde, além disso, extrapola as determinações biológicas focadas na doença. Este documento destaca a importância de incluir a sociedade na busca pela melhoria de sua saúde e qualidade de vida, a partir da identificação das próprias necessidades (HAESE et al., 
2012). Este documento indica campos de ações centrais para promover saúde, elaboração e implementação de políticas públicas, criação de ambientes favoráveis à saúde, incentivos às ações comunitárias, desenvolvimento de habilidades pessoais em consonância com o sistema de saúde (CONASS, 2016).

\section{CONCLUSÃO}

A educação em saúde tem sido largamente incentivada pelo Ministério da Saúde, pois se trata de uma forma de promover a saúde e estimular a troca de conhecimento entre setores, trazendo ganhos importantes para a população. A finalidade de ações educativas é desenvolver no indivíduo e no grupo a capacidade de analisar criticamente a sua realidade, de decidir ações conjuntas para resolver problemas e modificar situações, de organizar e realizar a ação e avaliá-la globalmente.

O presente trabalho não só corrobora com os achados literários, como também levanta questões de extrema importância para o bem estar da comunidade assim como de toda população de países em desenvolvimento. Os resultados obtidos nesse estudo, destacando as condições higiênico sanitárias precárias da população, demonstram a necessidade que a educação em saúde seja adotada como medida profilática. Sugere-se com esse trabalho que novos estudos com enfoque em diagnóstico e tratamento sejam realizados como medida de controle epidemiológico.

Os dados encontrados auxiliam na compreensão de como os fatores socioeconômicos e ambientais encontram-se interligados ao risco de contaminação, susceptibilidade e prevalência de doenças infecciosas e parasitárias, ressaltando a importância da educação em saúde com ênfase no autocuidado, higiene pessoal e saneamento básico.

\section{REFERÊNCIAS}

BIRCH D. A. Improving Schools, Improving School Health Education, Improving Public Health: The Role of SOPHE Members. Health Education Behavior, v. 44, n. 6, p. 839-844, 2017. Disponível em: <http://dx.doi.org/10.1177/1090198117736353>. doi: $10.1177 / 1090198117736353$.

BORGES, W. F.; MARCIANO, F. M.; OLIVEIRA, H. B. Parasitos intestinais: Elevada prevalência de Giardia lamblia em pacientes atendidos pelo serviço público de saúde da região sudeste de Goiás, Brasil. Revista de Patologia Tropical, v. 40, n. 2, p. 149-157, 2011. Disponível em: <https://doi.org/10.5216/rpt.v40i2.14940>. doi: 10.5216/rpt.v40i2.14940.

BROWN D.; FARROW C.; MCBEAN E. A.; GHARABAGHI B.; BEAUCHAMP J. Advancing performance evaluation standards for household water treatment technologies. Journal of Water and Health, v. 17, n. 2, p. 266-273, 2019. Disponível em: <https://doi.org/10.2166/wh.2018.266> doi: 10.2166/wh.2018.266.

CHAOUA S.; BOUSSAA S.; KHADRA A.; BOUMEZZOUGH A. Efficiency of two sewage treatment systems (activated sludge and natural lagoons) for helminth egg removal in Morocco. Journal of Infectious and Public Health, v. 11, n. 2, p. 197202, 2018. Disponível em: <https://doi.org/10.1016/j.jiph.2017.07.026> doi: 10.1016/j.jiph.2017.07.026. 
COATES S. J.; THOMAS C.; CHOSIDOW O.; ENGELMAN D.; CHANG A. Y. Ectoparasites: Pediculosis and tungiasis. Journal of the American Academy of Dermatology, v. 82, n. 3, p. 551-569, 2020. Disponível em: <https://doi.org/10.1016/j.jaad.2019.05.110> doi: 10.1016/j.jaad.2019.05.110.

CONASS. Promoção da Saúde Propostas do Conselho Nacional de Secretários de Saúde (CONASS) para sua efetivação como política pública no Brasil. Conselho Nacional de Secretários de Saúde, 2016. Disponível em: http://www.conass.org.br/wp-content/uploads/2016/06/CONASS.pdf.

DACAL E.; KÖSTER P. C.; CARMENA D. Diagnóstico molecular de parasitosis intestinales. Enfermedades Infecciosas y Microbiología Clínica, v. 38, n. 1, p. 2431, 2020. Disponível em: <https://doi.org/10.1016/j.eimc.2020.02.005> doi: 10.1016/j.eimc.2020.02.005.

DE CARLI, G. A.; TASCA, T.; MACHADO, A. R. L. Parasitoses Intestinais. In: DUNCAN, BB; SCHMIDT, MI \& GIUGLIANI, ERJ. Medicina Ambulatorial: condutas e atenção primária baseadas em evidência. 4. ed. Porto Alegre: Artmed, p. 1465-1475. 2013.

FELEKE B. E.; BEYENE M. B.; FELEKE T. E.; JEMBER T. H.; ABERA B. Intestinal parasitic infection among household contacts of primary cases, a comparative crosssectional study. PLoS One, v. 14, n. 10, 2019. Disponível em: <http://dx.doi.org/10.1371/journal.pone.0221190. doi: 10.1371/journal.pone.0221190.

FUNDAÇÃO NACIONAL DE SAÚDE. Manual de Orientações Técnicas para Elaboração e Apresentação de Propostas e Projetos para Sistemas de Esgotamento Sanitário. Fundação Nacional de Saúde. Brasília: Ministério da Saúde, 2019. Disponível em: http://www.funasa.gov.br/bibliotecaeletronica/publicacoes/engenharia-de-saude-publica

GOMES F. M. D. S.; SANTO M. C. C. D. E.; GRYSCHEK R. C. B.; BERTOLOZZI M. R.; FRANÇA F. O. S. Access to drinking water and sewage treatment in Brazil: a challenge for the control of waterborne infectious diseases. Revista do Instituto de Medicina Tropical de São Paulo, v. 30, n. 62, 2020. Disponível em: <http://dx.doi.org/10.1590/S1678-9946202062071>. doi: 10.1590/S16789946202062071.

HAESE, L. M.; BUCHELE, F.; BRZOZOWSKI, F. S. Considerações sobre a autonomia e a promoção da saúde. Physis Revista de Saúde Coletiva, v. 22, n. 2, p. 605-20, 2012. Disponível em: <https://doi.org/10.1590/S010373312012000200011 >. doi: 10.1590/S0103-73312012000200011.

HALLIDAY F. W.; PENCZYKOWSKI R. M.; BARRÈS B; ECK J. L.; NUMMINEN E; LAINE A. L. Facilitative priority effects drive parasite assembly under coinfection. Nature Ecology and Evolution, v. 4, n. 11, p. 1510-1521, 2020. Disponível em: <http://dx.doi.org/10.1038/s41559-020-01289-9>. doi: 10.1038/s41559-020-01289-9.

IBGE. Instituto Brasileiro de Geografia e Estatística. Pesquisa Nacional por Amostra de Domicílios Contínua. Características gerais dos domicílios e dos 
moradores. Brasil: IBGE, 2019. Disponível em: <https://biblioteca.ibge.gov.br/visualizacao/livros>

MADIGAN, M. T., MARTINKO, J. M.; PARKER, J.; Microbiologia de Brock. 14 ed. São Paulo: Artmed, 2016. p. 624.

MOLYNEUX, D. H.; SAVIOLI, L.; ENGELS, D. Neglected tropical diseases: progress towards addressing the chronic pandemic. Lancet, v. 389, n. 10066, 2017. Disponível em: <http://dx.doi.org/10.1016/S0140-6736(16)30171-4> doi: 10.1016/S0140-6736(16)30171-4.

MORAES, L. J. R.; ANDRADE, L. S.; FARIAS, C. B. P.; PINTO, L. C. Prevalência de anemia associada a parasitoses intestinais no território brasileiro: uma revisão sistemática. Revista Pan-Amazônica de Saúde, Ananindeua, v. 10, 2019. Disponível em: <http://dx.doi.org/10.5123/s2176-6223201900098>. doi: 10.5123/s2176-6223201900098.

NEVES, D. P. Parasitologia Humana. 13. ed. São Paulo: Atheneu, 2016.

RAY, B. Fundamental food microbiology. 5. ed. Boca Raton: CRC Press, p.608.2013.

REY, L. Flagelados das vias digestivas e geniturinárias: tricomoníase e giardíase. In: Bases da Parasitologia Médica. 3. ed. Rio de Janeiro: Guanabara Koogan, p. 424.2015.

SAPS - Secretaria de atenção primária a saúde. Política Nacional de Atenção Básica. Brasília: Ministério da Saúde. 2012. Disponível em: $<$ https://aps.saude.gov.br>

SHRESTHA A; SCHINDLER C; ODERMATT P; GEROLD J; ERISMANN S; SHARMA S; KOJU R; UTZINGER J; CISSÉ G. Intestinal parasite infections and associated risk factors among schoolchildren in Dolakha and Ramechhap districts, Nepal: a cross-sectional study. Parasites and Vectors. v. 11, n. 1, 2018. Disponível em: <https://doi.org/10.1186/s13071-018-3105-0>. doi: 10.1186/s13071-018-3105-0.

SILVA N.C.D.C.D.; MEKARO K.S.; SANTOS R.I.O.; UEHARA S.C.D.S.A. Knowledge and health promotion practice of Family Health Strategy nurses. Revista Brasileira de Enfermagem, v. 73, n. 5, 2020. Disponível em: <https://doi.org/10.1590/00347167-2019-0362>. doi: 10.1590/0034-7167-2019-0362.

WHO - World Health Organization. Neglected tropical disease. 2020. Disponível em: $<$ https://www.who.int/neglected_diseases/en/>. 